\title{
Design Participativo na Comunidade Brasileira de Informática na Educação: um mapeamento sistemático
}

\author{
Daniel Domingos Alves ${ }^{12}$, Jean C. S. Rosa² e Ecivaldo Matos ${ }^{2}$ \\ ${ }^{1}$ Instituto Federal de Mato Grosso (IFMT) - Campus Rondonópolis \\ Rondonópolis - MT - Brasil \\ 2Departamento de Ciência da Computação - Universidade Federal da Bahia (UFBA) \\ Salvador - BA - Brasil \\ \{daniel.domingos, jean.rosa, ecivaldo\}@ufba.br
}

\begin{abstract}
Participatory Design (PD) aims to promote the people active participation in the construction of artifacts, processes, and environments that shape their lives. PD can be a strong allied with Informatics in Education since it is the (potential) users (teachers, students) who must describe the most important criteria related to the need and desires for the construction of educational software. To map how the PD has been used by the Brazilian scientific community of Informatics in Education we conduct a systematic mapping of the literature and identified 11 papers on the subject. With the mapping, it was possible to show few studies that used PD in the context of Informatics in Education. Nevertheless, the studies found have important contributions when using PD in Informatics in Education.
\end{abstract}

Resumo. $O$ Design Participativo (DP) tem o objetivo de promover a participação ativa das pessoas na construção de artefatos, processos e ambientes que moldam a sua vida. O DP pode ser um forte aliado à Informática na Educação, pois quem deve descrever os critérios mais importantes relacionados à necessidade e desejos para a construção de um software educacional são os seus (potenciais) usuários (professores, estudantes). Para mapear como o DP tem sido utilizado pela comunidade científica brasileira de Informática na Educação, foi realizado um mapeamento sistemático da literatura que identificou 11 artigos sobre a temática. Com o mapeamento foi possível evidenciar que há poucos estudos que utilizaram DP no contexto de Informática na Educação. Apesar disso, os estudos encontrados apresentam importantes contribuições quando utilizado DP em Informática na Educação.

\section{Introdução}

O Design Participativo (DP) tem o objetivo de promover a participação ativa das pessoas na construção de artefatos, processos e ambientes que moldam as suas vidas. Nesse sentido, Simonsen e Robertson (2012) destacam que o DP em sua essência é um processo de investigar, compreender, refletir, estabelecer, desenvolver e apoiar a 
VII Congresso Brasileiro de Informática na Educação (CBIE 2018)

Anais dos Workshops do VII Congresso Brasileiro de Informática na Educação (WCBIE 2018)

aprendizagem e colaboração mútua entre os vários participantes da construção de artefatos (computacionais ou não).

Participação é o fundamento do DP [Simonsen e Robertson, 2012]. A participação de usuários na construção de artefatos pode ser dividida em quatro papéis: (i) usuário, (ii) testador, (iii) informante e (iv) parceiro de design [Druin, 2002]. Segundo Druin (2002), a participação do usuário no papel de usuário é limitada a observação das suas atividades cotidianas pelos designers; por sua vez, o usuário enquanto testador tem o objetivo de apreciar o artefato construído e relatar a sua experiência; o usuário no papel informante responde questões sobre os artefatos que já possuem ou sobre o seu cotidiano por meio de (mas não limitado à) entrevistas ou questionários; por fim, o usuário no papel de parceiro de design pode também assumir os papéis de usuário, testador e informante, e para além disso, é considerado como co-designer, podendo participar da tomada de decisão com a mesma relevância de um designer. Para Simonsen e Robertson (2012), o usuário como parceiro de design é a forma genuína e ativa da participação pregada pelo DP.

De acordo com Melo et al. (2012), o DP pode ser um forte aliado quando se trata da construção de softwares educacionais, isso porque, segundo Matos (2013), quem pode descrever os critérios mais importantes relacionados à necessidade e desejos para a construção de um software educacional são os seus usuários (professores, estudantes). Além disso, Rosa e Matos (2015) destacam ainda que o software educacional deve atender ao mais variado público, com diferenças culturais, sociais, cognitivas, de valores (etc.), subjetivas aos humanos e que, portanto, devem ser consideradas durante a modelagem de softwares, principalmente softwares educacionais [Rosa e Matos 2016].

Segundo os Grandes Desafios da Pesquisa em Computação no Brasil, existem barreiras tecnológicas, educacionais, culturais, sociais e econômicas que dificultam o acesso dos brasileiros ao conhecimento [Carvalho et al. 2006]. Por sua vez, os Grandes Desafios em Interação Humano-Computador no Brasil destacam a necessidade da construção de interfaces acessíveis, flexíveis e ajustáveis para favorecer o acesso participativo e universal ao conhecimento e afirmam que o desenvolvimento participativo de tecnologias digitais ainda é um desafio [Baranauskas, De Souza e Pereira 2012]. Os Grandes Desafios de Pesquisa em Sistemas de Informação no Brasil reforçam a complexidade na construção de softwares e confirmam a necessidade de metodologias robustas que estimulem e suportem a participação dos brasileiros [Araujo, Maciel e Boscarioli 2016].

Nesse sentido, problematiza-se a seguinte questão norteadora de pesquisa: como o DP tem sido utilizado pela comunidade científica brasileira de Informática na Educação?

Neste contexto, um mapeamento sistemático da literatura, baseado em Wohlin et al. [2012], foi conduzido com o objetivo de identificar como a comunidade científica brasileira de Informática na Educação tem utilizado o DP. A principal contribuição deste estudo é proporcionar aos pesquisadores e à comunidade de Informática na Educação informações que possam contribuir para a condução de novas pesquisas e na criação/adaptação de soluções de DP para o contexto de Informática na Educação. 
VII Congresso Brasileiro de Informática na Educação (CBIE 2018)

Anais dos Workshops do VII Congresso Brasileiro de Informática na Educação (WCBIE 2018)

\section{Metodologia}

O mapeamento sistemático foi realizado com base no modelo de protocolo ${ }^{1}$ apresentado por Wohlin et al. (2012), nas recomendações fornecidas por Kitchenham e Charters (2007).

\subsection{Questão e Subquestões de Pesquisa}

O mapeamento sistemático busca responder a seguinte questão de pesquisa:

\section{Como o Design Participativo tem sido utilizado pela comunidade científica brasileira de Informática na Educação?}

A questão de pesquisa principal foi decomposta nas seguintes subquestões de pesquisa mais detalhadas:
sub-QP1 - Quais estudos relatam experiência em utilizar DP em Informática na Educação?
sub-QP2 - Quais são os papéis dos participantes do DP? Como ocorre a seleção dos participantes?
sub-QP3 - Quais soluções (métodos, técnicas, modelos, ferramentas) de DP foram utilizadas elou propostas?

\subsection{Processo de Seleção de Estudos}

O processo do mapeamento sistemático de literatura compreende as seguintes etapas: (i) busca de estudos nas bases de dados; (ii) seleção de estudos ${ }^{2}\left(1^{\circ}\right.$ filtro: leitura de título e abstract e $2^{\circ}$ filtro: leitura completa dos estudos selecionados no $1^{\circ}$ filtro); (iii) extração de dados dos estudos incluídos; (iv) tabulação e análise dos estudos selecionados restantes.

\subsection{Estratégia de Busca}

Para a condução deste mapeamento sistemático, definiu-se a busca de estudos publicados em 4 periódicos e 5 anais de eventos científicos brasileiros que tratam do tema Informática na Educação. Foram considerados os estudos disponíveis nos sites dos periódicos da Revista Informática na educação: teoria \& prática, Revista Novas Tecnologias na Educação (RENOTE), Revista de Informática Teórica e Aplicada (RITA) e no portal da Comissão Especial de Informática na Educação (CEIE) da Sociedade Brasileira de Computação (SBC), o qual armazena as publicações da Revista Brasileira de Informática na Educação (RBIE) e anais dos seguintes eventos científicos: Simpósio Brasileiro de Informática na Educação (SBIE); Workshop de Informática na Escola (WIE); Workshops do Congresso Brasileiro de Informática na Educação (WCBIE); Jornada de Atualização em Informática na Educação (JAIE); Workshop de Desafios da Computação Aplicada à Educação (DesafIE).

\footnotetext{
${ }^{1} \mathrm{O}$ protocolo deste mapeamento sistemático está disponível em: http://bit.ly/WParTMapeamentoDP.

${ }^{2}$ Os critérios de inclusão e exclusão dos estudos são informados no protocolo de pesquisa.
} 
VII Congresso Brasileiro de Informática na Educação (CBIE 2018)

Anais dos Workshops do VII Congresso Brasileiro de Informática na Educação (WCBIE 2018)

Esses veículos de publicação foram escolhidos por possuírem grande parte das publicações sobre Informática na Educação no Brasil e devido ao acesso livre e gratuito aos periódicos e anais.

O processo de busca foi conduzido no portal da CEIE e nos sites dos periódicos Revista Informática na educação: teoria \& prática, RENOTE e RITA, por meio da ferramenta de busca dos sites.

As seguintes palavras-chave foram utilizadas: "participatory design", "design participativo", "participação do usuário", "user participation", "envolvimento do usuário", "user involvement".

De acordo com as palavras-chave, foi definida a string utilizada nas buscas, conforme a seguir:

("design participativo" OR "participatory design" OR "participação do usuário" OR "user participation" OR "envolvimento do usuário" OR "user involvement")

\section{Resultados}

Com a aplicação do protocolo do mapeamento sistemático, obteve-se os resultados que constam na Tabela 1. A extração de estudos das bases de dados foi realizada em agosto de 2018 e consistiu da aplicação da string de busca nas ferramentas de busca do portal da CEIE e dos sites dos periódicos Informática na educação: teoria \& prática, RENOTE e RITA.

Tabela 1: Resultados das etapas de busca de estudos, seleção preliminar e seleção de estudos

\begin{tabular}{|l|r|r|r|}
\hline Periódico/Conferência & BuscaBD & SelecPre & SelecEst \\
\hline RBIE & 5 & 4 & 3 \\
\hline SBIE & 6 & 4 & 2 \\
\hline WCBIE & 5 & 3 & 3 \\
\hline WIE & 2 & 2 & 1 \\
\hline JAIE & 1 & 0 & 0 \\
\hline DesafIE & 1 & 1 & 1 \\
\hline Informática na educação: teoria \& prática & 1 & 1 & 1 \\
\hline RENOTE & 1 & 1 & 0 \\
\hline RITA & 0 & 0 & 0 \\
\hline Total & $\mathbf{2 2}$ & $\mathbf{1 6}$ & $\mathbf{1 1}$ \\
\hline
\end{tabular}

BuscaBD: número de estudos retornados pelas buscas nas bibliotecas digitais; PreEst: número de artigos da seleção preliminar; SelecEst: número de artigos da seleção de estudos.

Com a aplicação da string de busca (citada na Seção 2) foram encontrados 22 estudos, separados por fonte, da seguinte forma: Revista Informática na educação: teoria \& prática, 1 estudo; RENOTE, 1 estudo; RITA, nenhum estudo; portal da CEIE, 20 estudos, sendo: RBIE, 5 estudos; SBIE, 6 estudos; WCBIE, 5 estudos; WIE, 2 estudos; JAIE, 1 estudo; DesafIE, 1 estudo.

\subsection{Seleção de estudos}

Nesta etapa, inicialmente foram lidos títulos e abstracts dos estudos encontrados na etapa de busca de estudos. Com base na leitura, e de acordo com os critérios de inclusão 
VII Congresso Brasileiro de Informática na Educação (CBIE 2018)

Anais dos Workshops do VII Congresso Brasileiro de Informática na Educação (WCBIE 2018)

e exclusão estabelecidos, 16 artigos foram selecionados para a próxima etapa e 6 foram rejeitados. Após essa seleção preliminar, os artigos selecionados foram lidos integralmente. Dos 16, 11 artigos foram selecionados para a extração de dados.

\subsection{Resultados da Extração de Dados}

Nesta subseção apresenta-se os resultados gerais das respostas à questão de pesquisa principal e das subquestões de pesquisa.

\subsubsection{Estudos que relatam experiência em utilizar DP em Informática na Educação}

Com relação à resposta à sub-QP1, que está relacionada aos estudos que relatam a experiência em utilizar DP em Informática na Educação, a seguir apresenta-se os estudos selecionados e descreve-se o contexto no qual o estudo está inserido.

Arantes et al. (2012) descrevem os resultados de um projeto que teve a participação ativa da comunidade de uma escola pública de ensino fundamental para integração de laptops em ambientes escolares por meio de uma abordagem semioparticipativa.

O artigo de Ribeiro e Melo (2017) apresenta um método de desenvolvimento de tecnologias digitais com crianças focalizado na integração entre a Engenharia de Software e a Interação Humano-Computador. O método é composto por brainstorming, braindraw, storyboarding, mock-ups e histórias de usuário. Crianças foram as participantes da aplicação e, de acordo com as autoras, foi possível perceber a dificuldade de expressão de ideias na forma escrita, sendo a forma oral e o desenho os meios mais produtivos de obter sua contribuição.

Melo, Baranauskas e Soares (2008) também trabalham com crianças como parceiras de design e propõem um método para o design centrado na participação da criança. As autoras fazem a associação entre técnicas participativas e técnicas da Semiótica Organizacional e a técnica percurso cognitivo cooperativo. Para as autoras, o Design Participativo é uma ferramenta valiosa para possibilitar o diálogo dos designers com as crianças.

Por sua vez, os artigos de Matsunaga, Borges e Moraes (2013) e Matsunaga et al. (2012) tratam do uso do DP para a construção de um jogo digital educacional para tratar sobre a doença hemofilia. Em ambos, os participantes foram crianças que auxiliaram a modelagem da interface como parceiros de design. Flores et al. (2017) também tratam sobre o desenvolvimento de um jogo auxiliar ao processo de ensino concebido por alunos de ensino superior com a participação de alunos de ensino médio, baseado em conceitos de DP.

Braz et al. (2016) apresentam as experiências das fases iniciais de um processo de DP de interfaces de usuários tangíveis, que podem promover a educação inclusiva. Professores participaram como parceiros de design por meio da ferramenta participativa MakeyMakey, que auxilia na prototipação de interfaces tangíveis. Todavia, os pesquisadores destacam que a discussão e avaliação de protótipos, bem como a experiência dos participantes em design e interfaces tangíveis podem ser um desafio durante o processo de desenvolvimento do produto. 
VII Congresso Brasileiro de Informática na Educação (CBIE 2018)

Anais dos Workshops do VII Congresso Brasileiro de Informática na Educação (WCBIE 2018)

Costa (2017) relata o desenvolvimento de um aplicativo para auxiliar na interação docente-discente em sala de aula. O desenvolvimento do aplicativo contou com a participação de pessoas com paralisia cerebral.

Melo et al. (2012) relatam experiências no contexto de um subprojeto para promoção do uso da informática por estudantes e professores da Educação Básica. Dentre algumas ações realizadas, os autores descrevem uma ação para constituir um espaço virtual para construção e troca de conhecimento. Essa ação contou com a participação de professores e foi realizada por meio de práticas participativas.

Os estudos de Falcão et al. (2017) e Peres, da Silva Oliveira e Morais (2017) apresentam um projeto que foi organizado como uma Comunidades de Prática e aplicou técnicas de DP no contexto educacional. Destaca-se que nos estudos de Falcão et al. (2017) e Peres, da Silva Oliveira e Morais (2017) o principal objetivo em utilizar DP está relacionado à aprendizagem decorrente da participação dos alunos no processo de DP.

\subsubsection{Participantes de DP em Informática na Educação}

Com relação às respostas à sub-QP2, a qual está relacionada aos papéis e seleção dos participantes do DP, os resultados deste mapeamento evidenciam a participação de profissionais da educação, alunos, crianças, pais de alunos e pessoas com deficiência. $\mathrm{O}$ Gráfico 1 apresenta o perfil dos participantes do processo de DP nos estudos selecionados. Neles, crianças participaram do processo de design participativo em 4 estudos; alunos de graduação, em 3 estudos; alunos do ensino médio, em 3 estudos; professores, em 3 estudos; pessoas com paralisia cerebral, em 1 estudo; outros (pesquisadores, alunos de ensino fundamental, pais de alunos, estagiários da escola, gestores da escola, parceiros da secretaria municipal de educação e funcionários da escola), em 1 estudo. Ressalta-se que em parte dos estudos selecionados houve a participação de mais de um perfil de participante no processo de design participativo.

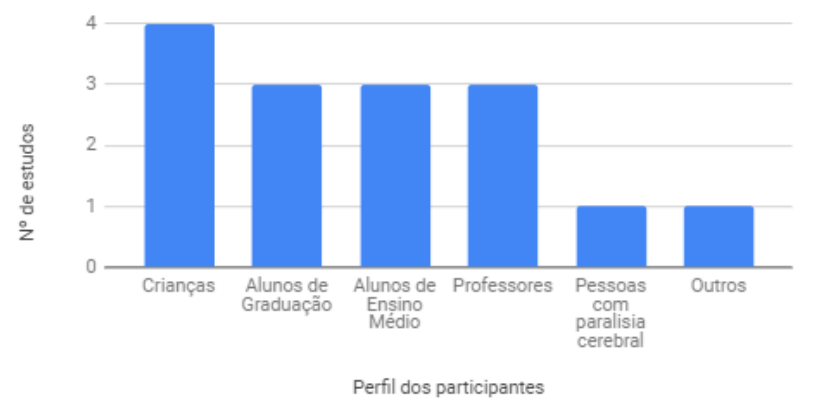

Gráfico 1: Perfis de Participantes do processo de DP.

$\mathrm{Na}$ maioria dos artigos selecionados [Arantes et al. 2012; Matsunaga, Borges e Moraes 201; Falcão et al. 2017; Matsunaga et al. 2012; Melo, Baranauskas e de Matos Soares 2008; Costa 2017; Peres, da Silva Oliveira e Morais 2017], os participantes foram envolvidos no DP como parceiros de design. Os estudos de Braz et al. (2016), Ribeiro e Melo (2017) e Melo et al. (2012) não descreveram explicitamente como foi efetivada a participação durante a realização de atividades de DP. Em Flores et al. (2017), considerou-se que os participantes foram informantes no processo de DP. 
VII Congresso Brasileiro de Informática na Educação (CBIE 2018)

Anais dos Workshops do VII Congresso Brasileiro de Informática na Educação (WCBIE 2018)

A seleção dos participantes foi descrita em apenas um dos estudos selecionados. Peres, da Silva Oliveira e Morais (2017) afirmam que os potenciais integrantes do projeto passaram por uma seleção constituída de duas etapas: questionário e dinâmica de grupo.

\subsubsection{Soluções de/para DP utilizadas ou propostas no contexto de Informática na Educação}

Com relação às respostas à sub-QP3, que está relacionada às soluções (métodos, técnicas, modelos, ferramentas) de DP utilizadas e/ou propostas, inicialmente foram analisadas se foram propostas novas técnicas, métodos ou processos de DP nos artigos selecionados. Os resultados deste mapeamento revelam que em apenas dois dos estudos selecionados [Arantes et al. 2012; Ribeiro e Melo 2017] foram propostas soluções para DP no contexto de Informática na Educação. Arantes et al. (2012) propuseram uma abordagem metodológica para a integração de laptops no ambiente escolar que tem base em conceitos e artefatos da Semiótica Organizacional e DP. Ribeiro e Melo (2017) propuseram um método de desenvolvimento de software por crianças utilizando o ambiente Scratch. No entanto, apenas o estudo de Arantes et al. (2012) apresenta avaliação/validação da solução.

Nos 11 estudos selecionados foram encontradas 26 soluções de/para DP utilizadas no contexto de Informática na Educação. O Gráfico 2 apresenta as soluções de/para DP mais utilizadas nos estudos selecionados. O braindraw e o brainstorming foram as soluções mais utilizadas.

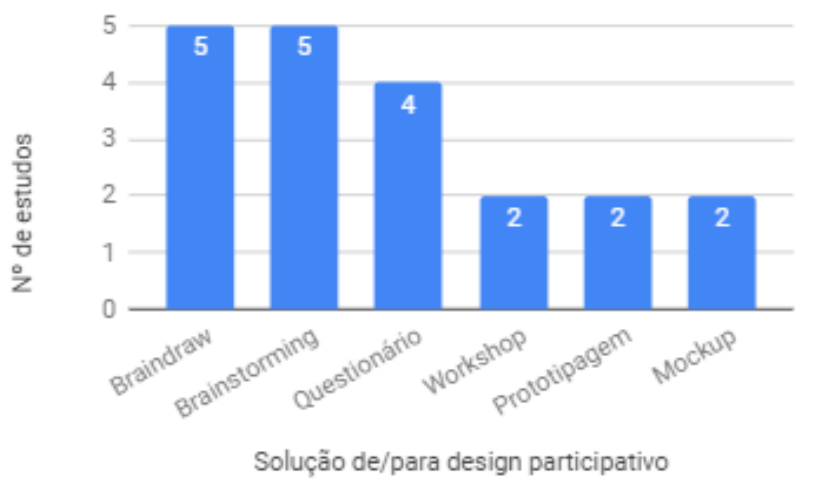

Gráfico 2: Soluções de/para DP mais utilizadas no contexto de Informática na Educação

\section{Análise}

Esta seção sumariza as principais considerações e discussões deste mapeamento sistemático, destacando as limitações que podem representar ameaças à sua validade.

As principais conclusões deste mapeamento são as seguintes:

- há poucos estudos primários sobre DP publicados em periódicos e anais de conferências de Informática na Educação no Brasil;

- em apenas dois estudos [Arantes et al. 2012; Ribeiro e Melo 2017] foram propostas soluções de/para DP no contexto de Informática na Educação. No entanto, apenas um dos estudos [Arantes et al. 2012] apresenta avaliação da 
VII Congresso Brasileiro de Informática na Educação (CBIE 2018)

Anais dos Workshops do VII Congresso Brasileiro de Informática na Educação (WCBIE 2018)

solução. Assim, verifica-se que há carência de soluções (abordagens, métodos, técnicas, ferramentas) de DP propostas para o contexto de Informática na Educação;

- o principal objetivo da maioria dos estudos selecionados está no desenvolvimento e/ou implantação de tecnologias educacionais. Em apenas dois estudos [Falcão et al. 2017; Peres, da Silva Oliveira e Morais 2017] de um mesmo projeto, o principal objetivo estava relacionado à aprendizagem de alunos no decorrer de sua participação durante as atividades de DP;

- Em apenas um estudo [Peres, da Silva Oliveira e Morais 2017] foi descrito de que forma ocorreu a seleção dos participantes.

Os resultados deste mapeamento sistemático sugerem a necessidade de um amplo apoio à comunidade de Informática na Educação pela comunidade de Interação Humano-Computador (IHC), por meio de esforços em pesquisas para a disponibilização de soluções de DP considerando as especificidades e necessidades educacionais.

Há, portanto, necessidade de fazer com que pesquisas e experiências de DP no contexto de Informática na Educação sejam desenvolvidas e publicadas. Por outro lado, pode-se mencionar também que há evidências de que DP pode contribuir para Informática na Educação. Apesar de poucos estudos, verifica-se resultados bem-sucedidos quando considerado DP no contexto de Informática na Educação. Diversas são as contribuições encontradas nos artigos selecionados, a citar:

- a participação ativa de usuários finais no projeto e/ou desenvolvimento de tecnologias educacionais;

- maior preocupação com os usuários;

- a utilização de DP para apoiar o processo de ensino-aprendizagem;

- participação de pessoas com deficiência no desenvolvimento de tecnologias educacionais;

- desenvolvimento de soluções de DP para o contexto de Informática na Educação.

Com o intuito de garantir um processo de seleção imparcial, todas as etapas do processo de mapeamento sistemático foram seguidas conforme o protocolo. Os autores realizaram a seleção dos estudos de forma independente e ao final, eventuais desacordos e dúvidas foram sanadas. Os veículos de publicações considerados contemplam grande parte dos principais periódicos e conferências de Informática na Educação no Brasil. No entanto, devido ao número limitado de base de dados utilizadas, é possível que estudos relevantes não tenham sido incluídos.

\section{Considerações Finais}

Este artigo apresentou um mapeamento sistemático que resume as informações existentes sobre a utilização de design participativo pela comunidade brasileira de Informática na Educação. O mapeamento sistemático selecionou 11 (onze) estudos primários.

O mapeamento sistemático identificou poucos estudos que utilizaram DP no contexto de Informática na Educação. Apesar disso, os estudos encontrados trouxeram importantes contribuições quando utilizado DP em Informática na Educação. 
VII Congresso Brasileiro de Informática na Educação (CBIE 2018)

Anais dos Workshops do VII Congresso Brasileiro de Informática na Educação (WCBIE 2018)

Enfim, enfatiza-se a relevância do tema e destaca-se a importância da utilização de DP pela comunidade de Informática na Educação. Espera-se que esse mapeamento tenha fornecido um panorama geral sobre os estudos que utilizaram DP no contexto de Informática na Educação e seja um esforço inicial para que novas pesquisas e propostas relacionadas a esse tema sejam realizadas.

\section{Agradecimentos}

Os autores agradecem o apoio da Coordenação de Aperfeiçoamento de Pessoal de Nível Superior (CAPES) pelo financiamento da pesquisa; e ao Grupo de Pesquisa e Extensão em Informática, Educação e Sociedade - Onda Digital (UFBA/CNPq). O primeiro autor ainda agradece ao Instituto Federal de Mato Grosso (IFMT).

\section{Referências}

Arantes, F. L., Amiel, T., de Miranda, L. C., Martins, M. C., and Baranauskas, M. C. C. (2012). Laptops Educacionais em Escolas Públicas: Primeiros Resultados de uma Abordagem Socio-Técnica. Revista Brasileira de Informática na Educação, 20(2), $31-43$.

Araujo, R. M. De, Maciel, R. S., and Boscarioli, C. (2016). I GranDSI-BR - Grandes Desafios de Pesquisa em Sistemas de Informação no Brasil 2016 a 2026. SBC. Porto Alegre: SBC.

Baranauskas, M. C. C., De Souza, C. S., and Pereira, R. (2012). I GranDIHC-BR Grandes Desafios de Pesquisa em Interação Humano-Computador no Brasil. SBC. Cuiabá: SBC.

Braz, L., Ramos, E., Benedetti, M. P., and Hornung, H. (2016). Design de tecnologia e educação inclusiva: explorando o espaço do problema. In: Proceedings of Brazilian Symposium on Computers in Education. p. 757-766.

Carvalho, A. C. P. L. et al. (2006). Grandes Desafios da Pesquisa em Computação no Brasil-2006-2016. SBC. Porto Alegre: SBC.

Costa, D. (2017). Desenvolvimento Participativo de Aplicativos para Pessoa com Paralisia Cerebral Utilizando Método DAPDA. In: Anais dos Workshops do Congresso Brasileiro de Informática na Educação. p. 1384-1393.

Druin, A. (2002). The role of children in the design of new technology. Behaviour \& Information Technology, 21(1), 1-25.

Falcão, T. P., Peres, F., Oliveira, G., and Morais, D. (2017). Design participativo de jogos educacionais na comunidade de prática do projeto DEMULTS. In: Anais dos Workshops do Congresso Brasileiro de Informática na Educação. p. 1344-1353.

Flores, G. L. M., Crestani, A. V., Bauer, R., Mombach, J., and Montanha, A. (2017). Design Participativo no Ensino Médio: Desenvolvimento de um Jogo Auxiliar ao Processo de Ensino. In: Anais dos Workshops do Congresso Brasileiro de Informática na Educação. p. 1324-1333.

Kitchenham, B. and Charters, S. (2007). Guidelines for performing systematic literature reviews in software engineering. In: Technical report, EBSE Technical Report. 
VII Congresso Brasileiro de Informática na Educação (CBIE 2018)

Anais dos Workshops do VII Congresso Brasileiro de Informática na Educação (WCBIE 2018)

Matos, E. de S. (2013). Dialética da Interação Humano-Computador: tratamento didático do diálogo midiatizado. Tese. Faculdade de Educação da Universidade de São Paulo - USP.

Matsunaga, R., Borges, M., and Moraes, R. (2015). Desenvolvimento de um jogo educativo para crianças com hemofilia. Revista Brasileira de Informática na Educação, 23(2), 59-69.

Matsunaga, R. M., Morais, S. N., Rossi, R. A., and Borges, M. A. (2012). Desenvolvimento de um jogo educativo para apoiar o aprendizado de pacientes com Hemofilia. In: Anais do Workshop de Desafios da Computação Aplicada à Educação p. 110-119.

Melo, A. M., Baranauskas, M. C. C., and de Matos Soares, S. C. (2008). Design com Crianças: da Prática a um Modelo de Processo. Revista Brasileira de Informática na Educação, 16 (1), 43-55.

Melo, A. M., Mombach, J. G., de Freitas Saldanha, J., and Wernz, M. C. G. (2012). Informática na educação: promovendo novos talentos no pampa. In: Anais do Workshop de Informática na Escola. p. s/p.

Melo, A. M., Saldanha, J. D. F., and Wernz, M. C. G. (2012). Desafios à pesquisa em Computação em contexto educacional - qualidade no uso de objetos de aprendizagem em perspectiva. In: Anais do Workshop de Desafios da Computação Aplicada à Educação. p. 60-69.

Peres, F. M. D. A., da Silva Oliveira, G., and de Morais, D. C. S. (2017). Os outros ocultos nas interfaces de jogos digitais educacionais. Informática na educação: teoria \& prática, 20.

Ribeiro, S., and Melo, A. (2017). Um Método para o Desenvolvimento de Software com Crianças Utilizando o Ambiente Scratch. In: Proceedings of Brazilian Symposium on Computers in Education. p. 1027-1036.

Rosa, J., and Matos, E. (2015). Considerando aspectos culturais no (re)design da interação de Ambientes Virtuais de Aprendizagem. In: Anais do Simpósio Brasileiro de Informática na Educação. p. 852-856.

Rosa, J. C. S., and Matos, E. (2016). Semio-Participatory Framework for Interaction Design of Educational Software. In: Proceedings of the 15th Brazilian Symposium on Human Factors in Computer Systems - IHC '16. p. s/p.

Simonsen, J., and Robertson, T. (2012). Routledge International Handbook of Participatory Design. Taylor \& Francis.

Wohlin, C., Runeson, P., Höst, M., Ohlsson, M. C., Regnell, B., and Wesslén, A. (2012). Experimentation in software engineering. Springer Science \& Business Media. 